\title{
Self-consistency in the phonon space of the particle-phonon coupling model
}

\author{
V. Tselyaev* and N. Lyutorovich \\ St. Petersburg State University, St. Petersburg 199034, Russia \\ J. Speth \\ Institut für Kernphysik, Forschungszentrum Jülich, D-52425 Jülich, Germany \\ P.-G. Reinhard \\ Institut für Theoretische Physik II, Universität Erlangen-Nürnberg, D-91058 Erlangen, Germany
}

(Received 12 December 2017; published 12 April 2018)

\begin{abstract}
In the paper the nonlinear generalization of the time blocking approximation (TBA) is presented. The TBA is one of the versions of the extended random-phase approximation (RPA) developed within the Green-function method and the particle-phonon coupling model. In the generalized version of the TBA the self-consistency principle is extended onto the phonon space of the model. The numerical examples show that this nonlinear version of the TBA leads to the convergence of results with respect to enlarging the phonon space of the model.
\end{abstract}

DOI: 10.1103/PhysRevC.97.044308

\section{INTRODUCTION}

The Green-function (GF) method is a powerful tool for solving the nuclear many-body problem (see Refs. [1,2]). General equations of this method are inherently nonlinear. However, in practical applications, the linearized versions of these equations are commonly used. In particular, the linearized Bethe-Salpeter equation for the response function is equivalent, with some additional assumptions, to the wellknown random-phase approximation (RPA). The nonlinearity in the nuclear-structure models is frequently contained only on the mean-field level where Hartree-Fock (HF) or Hartree-FockBogoliubov (HFB) approximations and the energy density functional (EDF) theory are applied. Nevertheless, several approaches were developed where effects of nonlinearity beyond the mean-field level were considered within the so-called self-consistent RPA (SCRPA, see Refs. [3-6]) and within the standard GF method in the Bethe-Salpeter and in the Dyson equations (see, e.g., Refs. [7-9]).

In the mean-field approach, e.g., in the RPA, the model space is restricted to one-particle-one-hole (1p1h) configurations. This is a reliable method to calculate energies and transition probabilities of low-lying collective states as well as the mean energies and total strengths of the high-lying giant resonances in even-even nuclei. For more details, like the spreading widths of giant resonances, one has to extend the configuration space by considering, e.g., two-particle-two-hole correlations within the second RPA (SRPA) [10-12], or by coupling phonons to the $1 \mathrm{p} 1 \mathrm{~h}$ configurations in a shell model approach or in a self-consistent way; see [13-17] and references therein. Another area of application of beyond-mean-field theories is

\footnotetext{
*tselyaev@mail.ru
}

the investigation of particle-phonon coupling and polarization effects in odd nuclei (see, e.g., Refs. [18-23]).

In Ref. [10], calculations of electric and charge-exchange resonances within SRPA were reported using a realistic twobody interaction. The power of such an approach can be seen in the prediction of the high-lying Gamow-Teller strength in heavy nuclei, which was experimentally detected many years later. More recently, similar calculations were published for the monopole resonance in ${ }^{16} \mathrm{O}$ [12] and giant electric resonances in some double magic nuclei [11] where different forces were used.

In the phonon-coupling models used so far the results depend on the number of phonons considered, and moreover the noncollective states may enter the phonon space that leads to a violation of the Pauli principle and to an overcounting problem (see [18,19]). Actually, the problem of the dependence of results on the number of phonons in these models is more serious because ultimately it leads to the ultraviolet divergence when the number of phonons indefinitely increases. This problem is aggravated in the models based on the Skyrme energy-density functionals in consequence of the velocitydependent terms appearing in the residual interaction and in the particle-phonon vertices (see, e.g., Ref. [24]). In a recent publication [25] we presented a scheme to select the most relevant phonons in order to avoid and mitigate these shortcomings to some extent. This model is a modification of the time blocking approximation (TBA) [26-28], which is one of the versions of the extended RPA developed within the GF method and the particle-phonon coupling model.

In the present paper we develop a nonlinear form of the TBA which we name the configuration blocking approximation (CBA). This approach is connected with the self-consistent determination of the phonon space of the model. It assumes some additional restrictions imposed on the phonons included in this space. In our study we focus on the analysis of the 
influence of these restrictions on the convergence of solutions of the model equations with respect to enlarging the phonon space.

In Sec. II the formalism of the nonlinear version of the TBA is introduced. In the first subsection II A we summarize the conventional self-consistent RPA which is the basis for all extended models. Our new approach in its general form is presented in subsection IIB. At first glance the equations look identical to the TBA equations. There is indeed only one decisive difference: The phonons which couple to the single-particle propagators are not the solutions of the RPA equation but the solutions of the TBA itself which introduces the nonlinearity. The first modification concerns the CBA which is presented in Sec. II C. Here we augment the nonlinear model with additional restrictions imposed on the phonon space. The reduced form of this nonlinear model is presented in Secs. IID and IIE. The method of construction of the phonon space of the model is described in Sec. IIF. In the short subsection IIG the relation between CBA and TBA is explicitly demonstrated. In Sec. III numerical results of our new approach are presented. We compare previous results with the present ones with emphasis on the dependence on the size of the phonon configuration space. The conclusions are given in the last section.

\section{THE FORMALISM OF NONLINEAR TBA}

\section{A. RPA as starting point and basis}

Before presenting the involved TBA theories, we summarize here the RPA, which serves as starting point for the further development and provides an appropriate basis of the description. Doing so, we introduce "en passant" also the basic notations used henceforth.

RPA determines the excitation spectrum of a many-body system in the $1 \mathrm{p} 1 \mathrm{~h}$ vicinity of the ground state. There are several ways to write the RPA equations. We use here a formulation in terms of the response operator $R^{\mathrm{RPA}} \equiv R_{12,34}^{\mathrm{RPA}}$, which plays the role of a one-body operator in $1 \mathrm{p} 1 \mathrm{~h}$ space (the numerical indices stand for the sets of the quantum numbers of some single-particle basis). This then reads

$$
\begin{aligned}
R^{\mathrm{RPA}}(\omega) & =-\left(\omega-\Omega^{\mathrm{RPA}}\right)^{-1} M^{\mathrm{RPA}}, \\
\Omega_{12,34}^{\mathrm{RPA}} & =\Omega_{12,34}^{(0)}+\sum_{56} M_{12,56}^{\mathrm{RPA}} V_{56,34}, \\
\Omega_{12,34}^{(0)} & =h_{13} \delta_{42}-\delta_{13} h_{42}, \\
M_{12,34}^{\mathrm{RPA}} & =\delta_{13} \rho_{42}-\rho_{13} \delta_{42}, \\
h_{12} & =\frac{\delta E[\rho]}{\delta \rho_{21}}, \\
V_{12,34} & =\frac{\delta^{2} E[\rho]}{\delta \rho_{21} \delta \rho_{34}},
\end{aligned}
$$

where $E[\rho]$ is the EDF of the model, the $\Omega$ are Hamiltonian matrices in $1 \mathrm{p} 1 \mathrm{~h}$ space (thus carrying four indices), $M^{\mathrm{RPA}}$ is the RPA norm matrix, $\rho$ is the single-particle density matrix, $h$ is the single-particle Hamiltonian, and $V$ is the residual interaction in the particle-hole channel. In the following we will also use the indices $p$ and $h$ to label the single-particle states of particles and holes, respectively, in the basis in which the density matrix $\rho$ and the Hamiltonian $h$ are diagonal (so that $h_{p p}=\varepsilon_{p}$ and $h_{h h}=\varepsilon_{h}$ ). The poles of $R^{\mathrm{RPA}}(\omega)$ determine the RPA spectrum of eigenfrequencies $\omega_{n}$.

The RPA response operator can be expressed explicitly in terms of the spectral representation

$$
R^{\mathrm{RPA}}(\omega)=-\sum_{n} \frac{\sigma_{n}\left|Z^{n}\right\rangle\left\langle Z^{n}\right|}{\omega-\omega_{n}},
$$

where $\sigma_{n}=\operatorname{sgn}\left(\omega_{n}\right)$ and the $\left|Z^{n}\right\rangle$ are the $n$th RPA eigenstates whose details are given by the explicit $1 \mathrm{p} 1 \mathrm{~h}$ expansion coefficients $Z_{12}^{n}$. Inserting the spectral expansion into the response equation (1a) yields the RPA equations explicitly in terms of the expansion coefficients:

$$
\sum_{34} \Omega_{12,34}^{\mathrm{RPA}} Z_{34}^{n}=\omega_{n} Z_{12}^{n} .
$$

Both forms, (1a) as well as (3), are used in practice for the practical solution of the RPA equations.

The entity of all RPA eigenstates constitutes an expansion in $1 \mathrm{p} 1 \mathrm{~h}$ space which is orthonormal,

$$
\left\langle Z^{n^{\prime}}\left|M^{\mathrm{RPA}}\right| Z^{n}\right\rangle=\delta_{n n^{\prime}} \operatorname{sgn}\left(\omega_{n}\right),
$$

and complete according to the closure relation

$$
\sum_{n} \sigma_{n}\left|Z^{n}\right\rangle\left\langle Z^{n}\right|=M^{\mathrm{RPA}} .
$$

The RPA basis does also allow a complete representation of matrix operators. Any matrix $A \equiv A_{12,34}$ in the $1 \mathrm{p} 1 \mathrm{~h}$ space can be written as

$$
\begin{aligned}
A & =\sum_{n n^{\prime}}\left|Z^{n}\right\rangle A_{n n^{\prime}}\left\langle Z^{n^{\prime}}\right|, \\
A_{n n^{\prime}} & =\sigma_{n} \sigma_{n^{\prime}}\left\langle Z^{n}\left|M^{\mathrm{RPA}} A M^{\mathrm{RPA}}\right| Z^{n^{\prime}}\right\rangle .
\end{aligned}
$$

\section{B. The nonlinear TBA equations}

The RPA equations were forcefully closed by the quasiboson approximation [29,30]. Releasing this restriction, they will couple to higher configurations which can be efficiently expanded in terms of the $1 \mathrm{p} 1 \mathrm{~h} \otimes$ phonon configuration, where "phonon" stands for a subset of RPA eigenstates which have large collective strength and so couple most strongly to the pure $1 \mathrm{p} 1 \mathrm{~h}$ states in the expansion. An explicit expansion in the full $1 \mathrm{p} 1 \mathrm{~h} \otimes$ phonon space is extremely costly because it includes too many unimportant contributions. The phonon-coupling models simplify that task by maintaining a $1 \mathrm{p} 1 \mathrm{~h}$ expansion while including the temporary detours through $1 \mathrm{p} 1 \mathrm{~h} \otimes$ phonon space by modifying the RPA interaction matrix. We use the phononcoupling model here in the form of the TBA [26-28]. In fact, we consider it here, in extension of previous applications, in its self-consistent form. This gives rise to a nonlinear equation for the eigenstates $v$ in terms of $1 \mathrm{p} 1 \mathrm{~h}$ coefficients $z_{12}^{v}$ and eigenfrequency $\omega_{v}$, which reads

$$
\sum_{34} \Omega_{12,34}^{\mathrm{CBA}}\left(\omega_{v}\right) z_{34}^{v}=\omega_{v} z_{12}^{v},
$$


with

$$
\begin{aligned}
& \Omega_{12,34}^{\mathrm{CBA}}(\omega)=\Omega_{12,34}^{\mathrm{RPA}}+\sum_{56} M_{12,56}^{\mathrm{RPA}} \bar{W}_{56,34}(\omega), \\
& \bar{W}_{12,34}(\omega)=W_{12,34}(\omega)-W_{12,34}(0) .
\end{aligned}
$$

The new ingredient in the nonlinear TBA (the superscript CBA in the matrix $\Omega^{\mathrm{CBA}}(\omega)$ will be explained in Sec. IIC) is the matrix $\bar{W}(\omega)$ which represents the induced interaction generated by the intermediate $1 \mathrm{p} 1 \mathrm{~h} \otimes$ phonon configurations. The subtraction of $W(0)$ in Eq. (6c) is necessary to avoid perturbation of the mean-field ground state [31,32] and to ensure stability of solutions of the TBA eigenvalue equation (see [33]). The key point is that $W(\omega)$ is expanded in terms of the $1 \mathrm{p} 1 \mathrm{~h} \otimes$ phonon configurations as

$$
\begin{aligned}
W_{12,34}(\omega) & =\sum_{c, \sigma} \frac{\sigma F_{12}^{c(\sigma)} F_{34}^{c(\sigma) *}}{\omega-\sigma \Omega_{c}}, \\
\Omega_{c} & =\varepsilon_{p^{\prime}}-\varepsilon_{h^{\prime}}+\omega_{\nu}, \quad \omega_{v}>0,
\end{aligned}
$$

where $\sigma= \pm 1, c=\left\{p^{\prime}, h^{\prime}, \nu\right\}$ is a combined index for the $1 \mathrm{p} 1 \mathrm{~h} \otimes$ phonon configurations, and $v$ is the phonon's index. We emphasize that these are the TBA phonons as they emerge from the fully fledged TBA equation. These phonons enter the induced interaction through the $F$ amplitudes,

$$
\begin{aligned}
F_{p h}^{c(+)} & =\delta_{p p^{\prime}} g_{h^{\prime} h}^{\nu}-\delta_{h^{\prime} h} g_{p p^{\prime}}^{\nu}, \\
g_{12}^{v} & =\sum_{34} V_{12,34} z_{34}^{v},
\end{aligned}
$$

which obey the symmetry relations

$$
F_{12}^{c(-)}=F_{21}^{c(+) *}, \quad F_{p h}^{c(-)}=F_{h p}^{c(+)}=0 .
$$

It is important to note that the larger expansion space which is implicitly contained in TBA has an impact on the normalization of the $z$ coefficients. The RPA norm (4a) is extended to

$$
\begin{gathered}
\left\langle z^{\nu}\left|M^{\mathrm{RPA}}-W_{\nu}^{\prime}\right| z^{\nu}\right\rangle=\operatorname{sgn}\left(\omega_{\nu}\right), \\
W_{\nu}^{\prime}=\left(\frac{d W(\omega)}{d \omega}\right)_{\omega=\omega_{\nu}} .
\end{gathered}
$$

The second term $\propto W_{v}^{\prime}$ is not connected with the nonlinearity of the TBA but arises already in the conventional TBA. It accounts for the probability carried over to the space of $1 \mathrm{p} 1 \mathrm{~h} \otimes$ phonon configurations. Correspondingly, the first term, covering the content of pure $1 \mathrm{p} 1 \mathrm{~h}$ states, is relatively reduced. These nonlinear TBA equations are self-consistent because the $z^{v}$ amplitudes and the energies $\omega_{v}$ which emerge from the eigenvalue equation (6a) are fed back into the phonon coupling amplitudes $F$ and the energies $\Omega_{c}$. This approach is thus superior to standard TBA and can be considered as the first iteration toward the full scheme. However, self-consistency involves subtle complications which inhibit immediate, naive solution of Eqs. (6) and (7). The problem is solved by configuration blocking outlined in the next subsection. This delivers at the same time a bonus: an unambiguous and very efficient rule for confining the intermediate states to the most relevant phonons.

\section{Configuration blocking approximation (CBA)}

In the nonlinear TBA described above, the following contradiction arises. Equations of the ordinary TBA include configurations of types $1 \mathrm{p} 1 \mathrm{~h}$ and $1 \mathrm{p} 1 \mathrm{~h} \otimes$ phonon, where the phonons are determined within the RPA. If we replace the RPA phonons in the matrix $W(\omega)$ by the solutions of the TBA equation (6a), the resulting equations will include implicitly configurations of types 3p3h and higher (see [26-28]). This will be reflected in the spectrum of the TBA solutions, which acquires the huge spectral density of complex configurations. Feeding this back into the induced interaction $W$ grows intractable. And, more importantly, it becomes contradictory as configurations of this type go beyond the framework of the TBA. Already at the level of the 1p1h configurations of RPA, we have to select the few most collective phonons to render TBA manageable and consistent (see Sec. IIF). But now we obtain a swarm of states which have more strength in higher configurations, i.e., in the $W^{\prime}$ term in the norm (9a), than in its $1 \mathrm{p} 1 \mathrm{~h}$ head. These states are clearly to be excluded. To formalize this decision, let us rewrite the normalization (9a) in the form

$$
\begin{aligned}
\left(z^{\nu}\right)_{\mathrm{RPA}}^{2}+\left(z^{\nu}\right)_{\mathrm{CC}}^{2} & =1, \\
\left(z^{\nu}\right)_{\mathrm{RPA}}^{2} & =\operatorname{sgn}\left(\omega_{\nu}\right)\left\langle z^{\nu}\left|M^{\mathrm{RPA}}\right| z^{\nu}\right\rangle, \\
\left(z^{\nu}\right)_{\mathrm{CC}}^{2} & =-\operatorname{sgn}\left(\omega_{\nu}\right)\left\langle z^{\nu}\left|W_{\nu}^{\prime}\right| z^{\nu}\right\rangle .
\end{aligned}
$$

The term $\left(z^{v}\right)_{\mathrm{RPA}}^{2}$ in Eq. (10a) represents the contribution of the $1 \mathrm{p} 1 \mathrm{~h}$ (RPA) components to the norm. The term $\left(z^{\nu}\right)_{\mathrm{CC}}^{2}$ represents the contribution of the complex configurations. It is obvious that all states with dominant $\left(z^{\nu}\right)_{\mathrm{CC}}^{2}$ must be discarded from the set of TBA phonons because they cannot contain any more sufficient collectivity to contribute to the induced interaction. To block these contributions which are dominated by complex configurations from entering the phonon space of TBA we impose the condition

$$
\left(z^{v}\right)_{\mathrm{RPA}}^{2}>\left(z^{v}\right)_{\mathrm{CC}}^{2}
$$

which together with Eq. (10a) means that

$$
\left(z^{\nu}\right)_{\mathrm{RPA}}^{2}>\zeta_{\min }^{2}, \quad \zeta_{\min }^{2}=\frac{1}{2} .
$$

We introduce the parameter $\zeta_{\min }^{2}$ to make the impact of the condition (11b) visible throughout the formalism. Only TBA states which satisfy Eq. (11b) will be included in the induced interaction of TBA. We refer to this model as the configuration blocking approximation (CBA). It is a combination of nonlinear TBA and norm blocking determined by Eq. (11b).

The blocking condition (11b) can be formulated in terms of a blocking factor $f_{v}$ which we have to introduce into Eq. (8a), which now reads as

$$
F_{p h}^{c(+)}=f_{\nu}\left(\delta_{p p^{\prime}} g_{h^{\prime} h}^{\nu}-\delta_{h^{\prime} h} g_{p p^{\prime}}^{\nu}\right) .
$$

To automatically embody the blocking condition we put

$$
f_{v}^{2}=\theta\left(\left(z^{v}\right)_{\mathrm{RPA}}^{2}-\zeta_{\mathrm{min}}^{2}\right),
$$

where $\theta$ is the Heaviside step function. Thus, in a sense, one can consider $f_{v}^{2}$ as an occupation number for the phonons.

At this point, however, the following difficulty arises. The TBA equations (6) and (7) combined with blocking condition 
(11b) pose a highly noninear problem. It is thus not guaranteed that a unique solution exists. In fact, one will find a couple of solutions. In the spirit of dominance of $1 \mathrm{p} 1 \mathrm{~h}$ states, we select as the most wanted solution the one which maximizes the total $1 \mathrm{p} 1 \mathrm{~h}$ content of the TBA active phonons [i.e., the phonons which enter the matrix $W(\omega)]$. We recall that $1 \mathrm{p} 1 \mathrm{~h}$ content is defined as the contribution $\left(z^{v}\right)_{\mathrm{RPA}}^{2}$ to the norm (9a). Thus we impose additionally the criterion that we select that TBA solution which yields

$$
\sum_{v_{a}}\left(z^{v_{a}}\right)_{\mathrm{RPA}}^{2}=\max ,
$$

where the summation runs over the TBA active phonons only, i.e., those states $v_{a}$ which obey condition (11b).

\section{CBA in diagonal approximation}

Even with the above discussed restrictions imposed on the space of the TBA phonons, the exact solution of the system of Eqs. (6), (7), (12), (8b), (8c), and (10b) remains a rather difficult task. To simplify these equations further we make use of a diagonal approximation to the induced interaction $\bar{W}(\omega)$. Similarly to the case of RPA, also the CBA response function

$$
R^{\mathrm{CBA}}(\omega)=-\left[\omega-\Omega^{\mathrm{CBA}}(\omega)\right]^{-1} M^{\mathrm{RPA}}
$$

has a spectral representation,

$$
R^{\mathrm{CBA}}(\omega)=-\sum_{v} \frac{\sigma_{v}\left|z^{v}\right\rangle\left\langle z^{v}\right|}{\omega-\omega_{v}},
$$

where $\sigma_{v}=\operatorname{sgn}\left(\omega_{v}\right)$. As stated in Sec. II A, any matrix $A$ in the $1 \mathrm{p} 1 \mathrm{~h}$ space can be written in terms of the RPA amplitudes $Z_{12}^{n}$ according to representation (5). We apply that to the CBA response matrix. First, we recast the definition (14a) of the response operator to a defining equation

$$
\left[\Omega^{\mathrm{CBA}}(\omega)-\omega\right] R^{\mathrm{CBA}}(\omega)=M^{\mathrm{RPA}},
$$

and write it explicitly as matrix equation in the basis of RPA states:

$$
\begin{gathered}
\sum_{n^{\prime \prime}}\left[\left(\omega-\omega_{n}\right) \delta_{n, n^{\prime \prime}}-\sigma_{n} \bar{W}_{n n^{\prime \prime}}(\omega)\right] R_{n^{\prime \prime} n^{\prime}}^{\mathrm{CBA}}(\omega)=-\sigma_{n} \delta_{n, n^{\prime}} \\
\bar{W}_{n n^{\prime}}(\omega)=\left\langle Z^{n}|\bar{W}(\omega)| Z^{n^{\prime}}\right\rangle .
\end{gathered}
$$

This equation now is solved in a diagonal approximation, yielding the approximate response

$$
\tilde{R}_{n n^{\prime}}^{\mathrm{CBA}}(\omega)=-\frac{\sigma_{n} \delta_{n, n^{\prime}}}{\omega-\omega_{n}-\sigma_{n} \bar{W}_{n n}(\omega)} .
$$

Furthermore, we note that the matrix element $\bar{W}_{n n}(\omega)$ is composed, according to Eqs. (7), of a sum of two terms:

$$
\bar{W}_{n n}(\omega)=\sum_{\sigma= \pm 1} \bar{W}_{n n}^{(\sigma)}(\omega)
$$

It is known already from RPA that the terms with negative frequency are very small for stable ground states [29], and only those situations are considered here. We assume that these negative-frequency contributions are not larger than off-diagonal terms, which are omitted in the diagonal approximations, and we neglect them altogether. According to Eq. (8c), this means that the term $\bar{W}_{n n}^{(\sigma)}(\omega)$ with $\sigma=-\sigma_{n}$ can be neglected. Thus we assume

$$
\bar{W}_{n n}(\omega)=\bar{W}_{n n}^{\left(\sigma_{n}\right)}(\omega)
$$

\section{E. Justification of the blocking value $\zeta_{\min }^{2}$}

The diagonal approximation simplifies the mathematical structure of the response poles to an extent that we can substantiate the choice $\zeta_{\min }^{2}=1 / 2$, as done in condition (11b), on formal grounds. For the following derivations, we employ the fact that the TBA corrections are small compared to the leading RPA structure, and label specifically $v \rightarrow(n, q)$, where $n$ stands for a certain RPA state which becomes the "bandhead" of the subsequent TBA structure and $q$ labels the many substates in the structure. Skipping the sum over $\sigma$ in Eq. (19) simplifies the structure of the response function $\tilde{R}_{n n}^{\mathrm{CBA}}(\omega)$ such that its poles $\tilde{\omega}_{n, q}$ become the roots of the equation

$$
\begin{aligned}
& \sigma_{n} \tilde{\omega}_{n, q}\left[1+\sum_{c} \frac{\left|\tilde{F}_{n}^{c\left(\sigma_{n}\right)}\right|^{2}}{\tilde{\Omega}_{c}\left(\tilde{\Omega}_{c}-\sigma_{n} \tilde{\omega}_{n, q}\right)}\right]=\left|\omega_{n}\right|, \\
& \tilde{F}_{n}^{c(\sigma)}=\sum_{12} Z_{12}^{n *} F_{12}^{c(\sigma)}, \\
& \tilde{\Omega}_{c}=\varepsilon_{p^{\prime}}-\varepsilon_{h^{\prime}}+\sigma_{n^{\prime}} \tilde{\omega}_{n^{\prime}, q^{\prime}}
\end{aligned}
$$

where we use the combined index $c \equiv\left(p^{\prime}, h^{\prime}, v^{\prime}=\left(n^{\prime}, q^{\prime}\right)\right)$. From that it follows that $\sigma_{n} \tilde{\omega}_{n, q}>0$ for all $n$ and $q$. Now, the pole expansion of the (diagonal) response (18) has the form

$$
\begin{aligned}
\tilde{R}_{n n^{\prime}}^{\mathrm{CBA}}(\omega) & =-\sum_{q} \frac{\sigma_{n} \zeta_{n, q}^{2} \delta_{n, n^{\prime}}}{\omega-\tilde{\omega}_{n, q}}, \\
\zeta_{n, q}^{2} & =\left[1+\sum_{c} \frac{\left|\tilde{F}_{n}^{c\left(\sigma_{n}\right)}\right|^{2}}{\left(\tilde{\Omega}_{c}-\sigma_{n} \tilde{\omega}_{n, q}\right)^{2}}\right]^{-1} .
\end{aligned}
$$

This allows us to derive a sum rule for the coefficients $\zeta_{n, q}^{2}$ by comparing the first terms of the expansions in powers of $1 / \omega$ of the right-hand sides of Eqs. (18) and (22a). Namely, we have for all $n$

$$
\sum_{q} \zeta_{n, q}^{2}=1
$$

From Eqs. (5a) and (22a) we obtain

$$
\tilde{R}^{\mathrm{CBA}}(\omega)=-\sum_{n, q} \frac{\sigma_{n} \zeta_{n, q}^{2}\left|Z^{n}\right\rangle\left\langle Z^{n}\right|}{\omega-\tilde{\omega}_{n, q}} .
$$

Comparing Eqs. (14b) and (24), we confirm that in the diagonal approximation we have $v=(n, q)$ and

$$
\left|z^{v}\right\rangle=\zeta_{n, q}\left|Z^{n}\right\rangle, \quad \omega_{v}=\tilde{\omega}_{n, q}, \quad \sigma_{\nu}=\sigma_{n} .
$$

From Eqs. (10b) and (25) it follows that in this case we have

$$
\left(z^{v}\right)_{\mathrm{RPA}}^{2}=\zeta_{n, q}^{2}
$$


Altogether, condition (11b) in the diagonal approximation takes the form

$$
\zeta_{n, q}^{2}>\zeta_{\min }^{2}=1 / 2
$$

Now we can finally argue in favor of the choice $\zeta_{\min }^{2}$ for the blocking criterion: From Eq. (23) we see that

(i) there exists not more than one pole of the function $\tilde{R}_{n n}^{\mathrm{CBA}}(\omega)$ for which $\zeta_{n, q}^{2}>1 / 2$, and

(ii) two and more poles of this function can satisfy the condition $\zeta_{n, q}^{2}>\zeta_{\min }^{2}$ in the case $\zeta_{\min }^{2}<1 / 2$.

Thus one can consider the value $\zeta_{\min }^{2}=1 / 2$ as a threshold below which the fragmentation of the RPA state $\left|Z^{n}\right\rangle$ becomes significant. If there are no poles of the function $\tilde{R}_{n n}^{\mathrm{CBA}}(\omega)$ with $\zeta_{n, q}^{2}>1 / 2$, then all the TBA fragments of the RPA state $\left|Z^{n}\right\rangle$ have a structure going beyond the $1 \mathrm{p} 1 \mathrm{~h}$ approximation. This conclusion corroborates the reasoning used in the derivation of the condition (11b).

In the calculations presented below, we use the CBA scheme in the diagonal approximation. In this scheme, the active TBA phonons (see Sec. IIC) are found from the solution of the system of equations (21) together with Eqs. (8b), (8c), (12a), (12b), (22b), and (25). According to the last equation, this scheme can be also called renormalized TBA. Equation (6a) is solved in CBA using the full $1 \mathrm{p} 1 \mathrm{~h}$ space, i.e., without diagonal approximation.

To solve the system (21), the following iterative procedure is employed. We start from a certain basis set of the RPA phonons determined by Eqs. (3) and (4a) and we abbreviate this basis set as $\mathfrak{B}_{\mathrm{RPA}}^{(0)}$. It is constructed according to the criterion described in Sec. IIF. The set of the active TBA phonons is determined iteratively and will be denoted as $\mathfrak{B}_{\mathrm{TBA}}^{(\alpha)}$, where $\alpha$ is the iteration counter. According to Eqs. (25), there is a one-to-one correspondence between the set $\mathfrak{B}_{\mathrm{TBA}}^{(\alpha)}$ and a subset of $\mathfrak{B}_{\mathrm{RPA}}^{(0)}$ which we denote as $\mathfrak{B}_{\mathrm{RPA}}^{(\alpha)}$. Both sets $\mathfrak{B}_{\mathrm{RPA}}^{(\alpha)}$ and $\mathfrak{B}_{\mathrm{TBA}}^{(\alpha)}$ are simultaneously optimized in the course of the iteration. In the very first iteration, $\mathfrak{B}_{\mathrm{TBA}}^{(1)}$ is simply a certain subset of $\mathfrak{B}_{\mathrm{RPA}}^{(0)}$. In a couple of early iterations, the system (21) is solved for all RPA phonons (which are indicated by the index $n$ ) from the set $\mathfrak{B}_{\mathrm{RPA}}^{(0)}$. But from a certain iteration on, we solve Eqs. (21) only for the phonons from the set $\mathfrak{B}_{\mathrm{RPA}}^{(\alpha)}$ determined in the previous iteration. Thus, starting from this iteration, the sets $\mathfrak{B}_{\mathrm{RPA}}^{(\alpha)}$ and $\mathfrak{B}_{\mathrm{TBA}}^{(\alpha)}$ can only decrease, because states excluded according to criterion (27) and to the correspondence (25) between phonons in $\mathfrak{B}_{\mathrm{RPA}}^{(\alpha)}$ and $\mathfrak{B}_{\mathrm{TBA}}^{(\alpha)}$ do not appear again in the set $\mathfrak{B}_{\mathrm{RPA}}^{(\alpha)}$. This provides eventually a convergent procedure.

\section{F. Construction of the space of the RPA phonons}

The strength of CBA is that it implies a natural criterion for the selection of those phonons which are active in the induced interaction $W$. There remains, nonetheless, an issue of efficiency. The diagonal approximation outlined in Secs. IID and IIE proceeds through a representation in terms of RPA phonons, and these have much different impact on $W$. Thus it is useful to restrict the summation to the most important phonons. This is, at a technical level, again the same quest to find the most collective phonons, as in standard TBA, and many different recipes are used in the literature using a phonon coupling model. In connection with standard TBA, we had introduced in Ref. [34] an efficient criterion for the selection of the collective RPA phonons. The idea is to take the average strength $\langle V\rangle_{n}$ of the RPA residual interaction in state $n$ as measure of collectivity. This is plausible because it is the residual interaction which mixes the pure $1 \mathrm{p} 1 \mathrm{~h}$ states to a coherent superposition of many states. Moreover, states with large $\langle V\rangle_{n}$ are generally strong coupling states and thus will also contribute dominantly to the induced interaction. Considering this strength relative to excitation energy then leads to the dimensionless measure of collectivity:

$$
\begin{aligned}
v_{n} & =\langle V\rangle_{n} /\left|\omega_{n}\right|, \\
\langle V\rangle_{n} & =\left\langle Z^{n}|V| Z^{n}\right\rangle \\
& =\sum_{p h}\left[\left(\omega_{n}-\varepsilon_{p h}\right)\left|Z_{p h}^{n}\right|^{2}-\left(\omega_{n}+\varepsilon_{p h}\right)\left|Z_{h p}^{n}\right|^{2}\right],
\end{aligned}
$$

where $\varepsilon_{p h}=\varepsilon_{p}-\varepsilon_{h}$. We include into the phonon basis of the TBA only the phonons with

$$
\left|v_{n}\right|>v_{\text {min }}
$$

for some given value of $v_{\min }$. This criterion had been tested extensively in [34]. Plotting the distribution of $v_{n}$, we found a clear threshold value of $v_{n}=0.05$ below which the distribution becomes rapidly diffuse, and we took this as a physically sound cutoff criterion.

However, the criterion has two mild drawbacks. First, taking simply the average residual interaction $\langle V\rangle_{n}$ overlooks a few collective states having strong coupling matrix elements which unfortunately happen to compensate for each other in the average. Second, the simple inverse energy weight gives much emphasis to high-energy phonons while we expect the strongest contributions to $W$ from the low-energy collective modes. This leads us to propose here an improved criterion based on the average of the square of the residual interaction in state $n$, which can be reduced to

$$
\begin{aligned}
\left\langle V^{2}\right\rangle_{n} & =\left\langle Z^{n}\left|V^{2}\right| Z^{n}\right\rangle \\
& =\sum_{p h}\left[\left(\omega_{n}-\varepsilon_{p h}\right)^{2}\left|Z_{p h}^{n}\right|^{2}+\left(\omega_{n}+\varepsilon_{p h}\right)^{2}\left|Z_{h p}^{n}\right|^{2}\right],
\end{aligned}
$$

where the Hermitean property $V^{\dagger}=V$ is taken into account. From this, we form the dimensionless quantity

$$
\kappa_{n}=\frac{\gamma_{n}^{2}}{1+\gamma_{n}^{2}}, \quad \gamma_{n}^{2}=\left\langle V^{2}\right\rangle_{n} / \omega_{n}^{2}
$$

as new measure for collectivity. Now we see that $\kappa_{n}=0$ requires that RPA eigenfrequency equals exactly one of the $\pm \varepsilon_{p h}$ and thus is strictly uncorrelated. Consequently, small values of $\kappa_{n}$ signal small collectivity, i.e., coupling strength, throughout. Moreover, the energy weight $\omega_{n}^{-2}$ yields the wanted weight on low-energy states. 
By noting that the amplitudes of the quasiparticle-phonon interaction $g_{12}^{n}$ in the RPA are defined as

$$
g_{12}^{n}=\sum_{34} V_{12,34} Z_{34}^{n}
$$

we obtain from Eqs. (29a) and (30)

$$
\left\langle V^{2}\right\rangle_{n}=\left\langle g^{n} \mid g^{n}\right\rangle
$$

In the macroscopic approach (see, e.g., Refs. $[13,35])$ the amplitudes $g_{12}^{n}$ are proportional to the deformation parameters $\beta_{n}$ of the respective vibrational modes. So, in this approach $\gamma_{n}^{2} \propto \beta_{n}^{2}$. This shows explicitly that the selection of the phonons with the largest values of $\gamma_{n}^{2}$ corresponds to the selection of the low-energy vibrational modes with the largest deformation parameters having the most strong coupling to the single-particle states.

Finally note that the states which are usually referred to as the collective vibrational modes $\left(3_{1}^{-}\right.$levels in ${ }^{16} \mathrm{O}$ and ${ }^{40} \mathrm{Ca}, 2_{1}^{+}$, $3_{1}^{-}$, and $4_{1}^{+}$levels in ${ }^{48} \mathrm{Ca}, 2_{1}^{+}, 3_{1}^{-}, 4_{1}^{+}, 5_{1}^{-}$, and $6_{1}^{+}$levels in ${ }^{208} \mathrm{~Pb}$ ) have $\gamma_{n}^{2} \gtrsim 1$ and, consequently, $\kappa_{n} \gtrsim 0.5$. These archetype collective modes set the benchmark for collectivity. States with $\kappa_{n} \approx 0.1$ are still acceptably strong phonons. Values $\ll 1$ signal noncollective states.

\section{G. Standard TBA as limit of CBA}

As stated above, standard TBA can be obtained as the first iteration of CBA. It amounts to replacing the TBA spectrum in Eqs. (7) for the induced interaction $W$ with the mere RPA spectrum, i.e., by simply identifying in the $1 \mathrm{p} 1 \mathrm{~h} \otimes$ phonon summations

$$
c^{\mathrm{CBA}}=\left\{p^{\prime}, h^{\prime}, \nu\right\} \longrightarrow c^{\mathrm{RPA}}=\left\{p^{\prime}, h^{\prime}, n\right\},
$$

where we emphasize the transition here symbolically through the upper indices CBA and RPA.

The discussion of cutoff criteria in the selection of RPA states (see previous section) is of particular importance for standard TBA. A proper selection of a few most collective states is compulsory in any phonon coupling model to avoid double counting of the complex configuration and violation of the Pauli principle.

\section{RESULTS AND DISCUSSION}

Before presenting the results, a few words about the calculations are in order. We have computed the same test cases with standard TBA and with CBA in comparison using the same numerical procedures, which are explained in detail in Refs. [16,25,34]. The maximum energy of the single-particle states of the $1 \mathrm{p} 1 \mathrm{~h}$ basis was taken as $100 \mathrm{MeV}$. For ${ }^{16} \mathrm{O}$ and ${ }^{40} \mathrm{Ca}$ we did not use an energy cutoff on the phonon space, while for ${ }^{208} \mathrm{~Pb}$ the phonon basis was restricted by a maximum phonon energy of $100 \mathrm{MeV}$. For all cases we use the Skyrme parametrization SV-m64k6 [36] which, due its low effective mass, provides a particularly critical test of the impact of stability and convergence.

It is standard practice to check the properties of standard TBA under variation of the cutoff parameter, $v_{n}$ or $\kappa_{n}$. This is not so obvious in CBA because the proper cutoff is set by

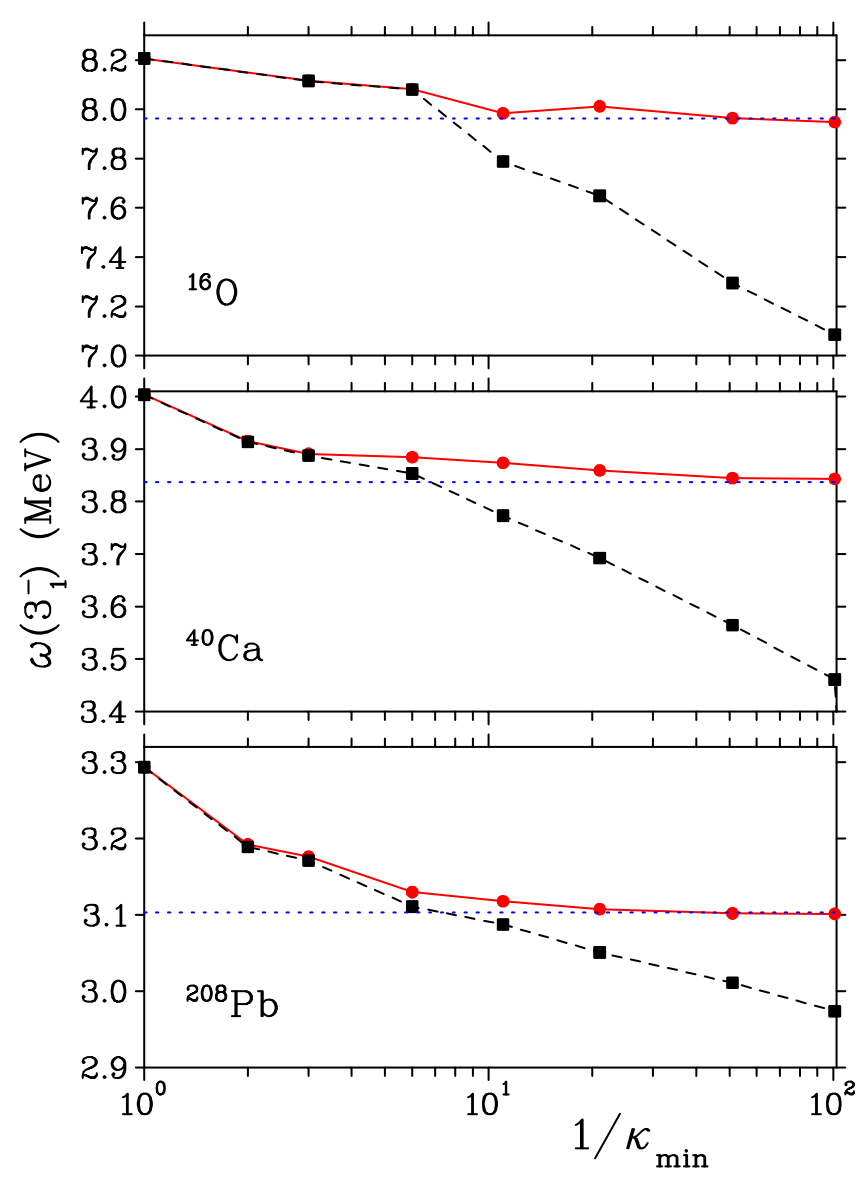

FIG. 1. Dependence of the energy of $3_{1}^{-}$state calculated in the CBA with $\zeta_{\min }^{2}=0.5$ (red solid lines) and in the standard TBA (black dashed lines) on the value of the inverse cutoff parameter $1 / \kappa_{\min }$. The fine dashed horizontal line indicates the energy as found in a previous paper using standard TBA with optimized cutoff parameter $v_{\text {min }}=0.05$ [34]. The experimental values are given in Table I.

theoretical considerations. Nonetheless, we use the same sort of cutoff in RPA space as a preselection of the expansion basis. Thus one can very well present results as a function of this preselection cutoff $\kappa_{\min }$ and so study convergence of the method. This is what we will do in several of the following figures.

In Fig. 1 we present the results of the energies of the first $3^{-}$ levels in ${ }^{16} \mathrm{O},{ }^{40} \mathrm{Ca}$, and ${ }^{208} \mathrm{~Pb}$. The value $\kappa_{\min }=1$ corresponds to the RPA. The CBA results show nice convergence with the expansion space $1 / \kappa_{\min }$, and the converged result agrees perfectly with the results of our previous analysis [34]. The standard TBA result, however, shows an almost constant slope (in $\operatorname{logarithmic} \kappa_{\min }$ scale). Here we need again a separate analysis of the distribution of $\kappa_{n}$ to find the optimum value of $\kappa_{n}$. Doing that, we find an optimum cutoff $\kappa_{\min }$ in the interval 0.11-0.16 which yields excitation energies again close to the previous results (fine dashed horizontal line) and CBA. The interesting message is that CBA comes to the correct result without a separate decision on cutoff parameters. Of course, one still wants to check convergence with $\kappa_{n}$. But this is a technical aspect.

In Fig. 2 the transition probabilities of the $3_{1}^{-}$states in these three nuclei are presented. The quantities are the most sensitive 


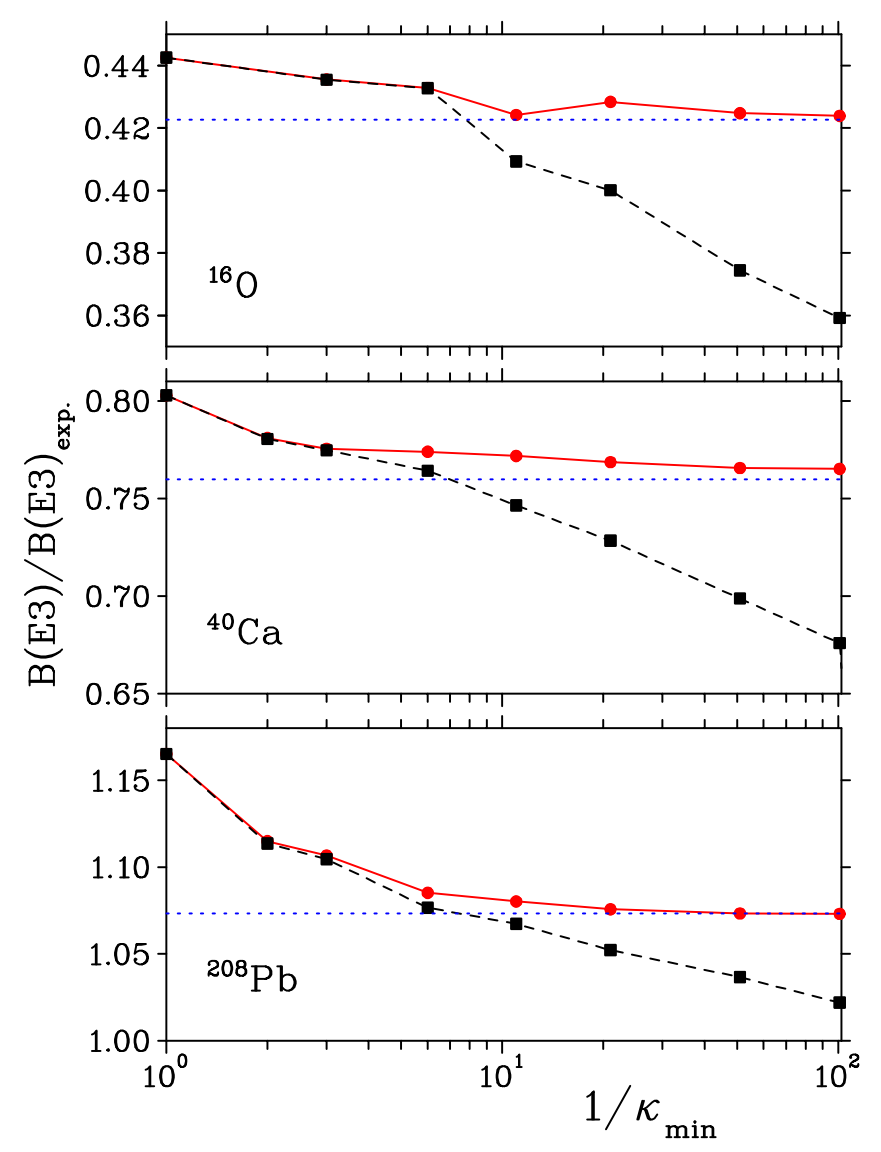

FIG. 2. Same as in Fig. 1 but for the reduced probabilities $B\left(E 3 ; 0_{\text {g.s. }}^{+} \rightarrow 3_{1}^{-}\right)$in units of their experimental values.

properties for any nuclear structure model as they are directly connected with the collective wave function. First of all one notices that the values converge within our newly developed theory whereas the conventional TBA results do not show this behavior. For ${ }^{208} \mathrm{~Pb}$ the agreement with data is very good, for ${ }^{40} \mathrm{Ca}$ fair, but for ${ }^{16} \mathrm{O}$ we are off by more then a factor of 2. Obviously in light nuclei one does not have enough $1 \mathrm{p} 1 \mathrm{~h}$ configurations for creating collective states.

Figure 3 shows the analogous results for the mean energies of the giant dipole resonance (GDR). The mean energies were defined as the values of the Lorentzian parameter $E_{0}$ determined by equating the energy-weighted moments of the calculated strength functions with the respective moments of the Lorentzian function. The moments were calculated within the following finite energy intervals whose centers approximately coincide with $E_{0}: 0-40 \mathrm{MeV}$ for ${ }^{16} \mathrm{O}, 10-30 \mathrm{MeV}$ for ${ }^{40} \mathrm{Ca}$, and $7-21 \mathrm{MeV}$ for ${ }^{208} \mathrm{~Pb}$. The trends, observations, and conclusions are exactly the same as in the previous figures.

The detailed distributions of the GDR strength distributions (the photoabsorption cross sections) in ${ }^{16} \mathrm{O},{ }^{40} \mathrm{Ca}$, and ${ }^{208} \mathrm{~Pb}$ are shown in Figs. 4-6. In all calculations of the GDR strength in ${ }^{16} \mathrm{O}$ and ${ }^{40} \mathrm{Ca}$ the single-particle continuum was included as described in [16]. The smearing parameters were $200 \mathrm{keV}$ in ${ }^{16} \mathrm{O}$ and $400 \mathrm{keV}$ in ${ }^{40} \mathrm{Ca}$ and ${ }^{208} \mathrm{~Pb}$. Standard TBA calculations which were done for comparison use the optimized cutoff parameter $v_{\min }=0.05$. The value $\kappa_{\min }=0.01$ was used in

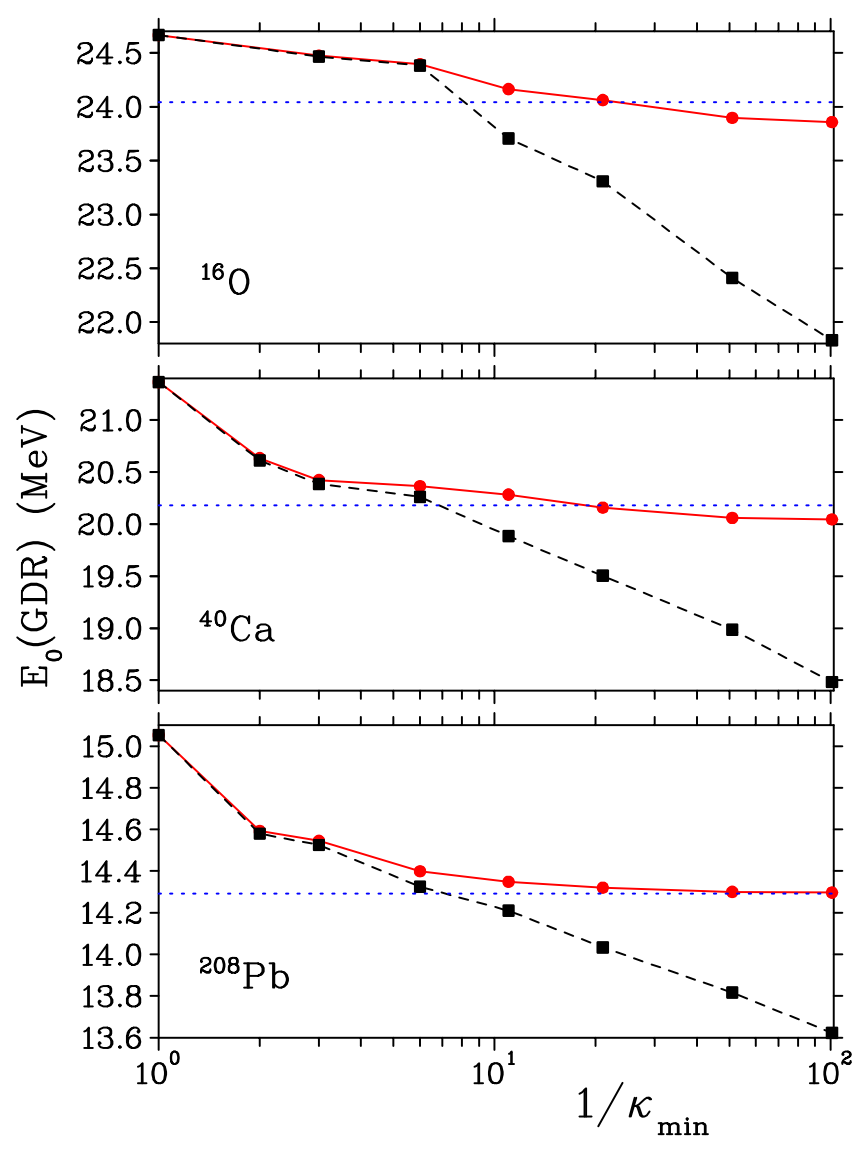

FIG. 3. Same as in Fig. 1 but for the mean energies (Lorentzian parameter $E_{0}$ ) of the giant dipole resonance.

CBA for limiting the RPA expansion basis, which is well on the safe side as we see from Figs. 1-3.

Figures 4-6 shows the same relative trends for all three nuclei. The spectra from CBA agree well with those of

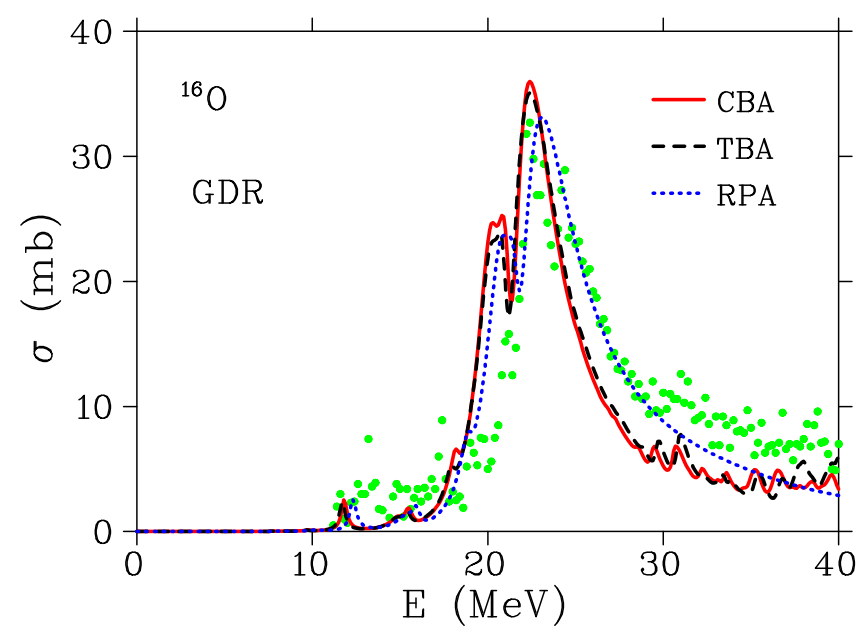

FIG. 4. The giant dipole resonance (GDR) in ${ }^{16} \mathrm{O}$ calculated in the CBA (red solid line), in standard TBA (black dashed line), and in RPA (blue dotted line). Experimental data from Ref. [37] are shown by the green circles. 


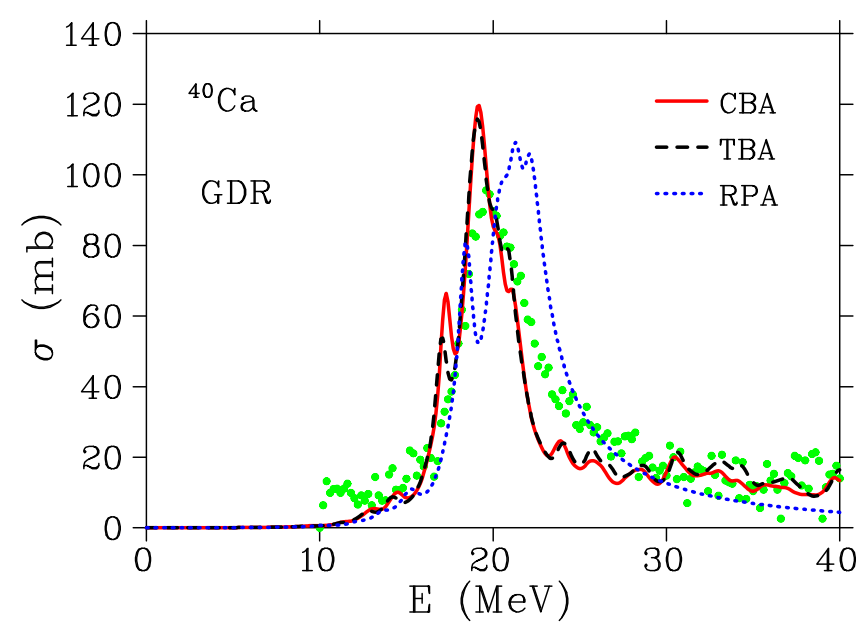

FIG. 5. Same as in Fig. 4 but for ${ }^{40} \mathrm{Ca}$. Experimental data are taken from Ref. [38].

standard TBA, and both differ significantly from RPA. TBA induces a small down-shift and, more important, smooths the spectra significantly. This smoothing is important to bring the theoretical distributions close to the experimental profile.

As can be seen from Figs. 1-3, the convergence in the CBA results is achieved at $\kappa_{\min } \approx \gamma_{\min }^{2}$ in the interval 0.02-0.05, which approximately corresponds to the boundary of the noncollective phonons as assessed by plotting the density of phonon states [34]. The results of the ordinary TBA do not show the tendency to convergence. The reason for the convergence in the CBA is a decrease of the number of phonons as compared with the ordinary TBA owing to an additional criterion for the selection of the phonons, Eq. (27), determined by the parameter $\zeta_{\min }^{2}$. This decrease becomes very strong when $\gamma_{\min }^{2}$ and $\kappa_{\min }$ are very small (see Fig. 7). At $0.02 \leqslant \gamma_{\min }^{2} \leqslant 0.05$ the number of active TBA phonons in the CBA is in the intervals 10-22 for ${ }^{16} \mathrm{O}, 24-45$ for ${ }^{40} \mathrm{Ca}$, and $77-105$ for ${ }^{208} \mathrm{~Pb}$. In practice, all the phonons with $\omega_{n}>\omega_{\max }$ for some value of $\omega_{\max }$ appear to be strongly fragmented; that is, they have $\zeta_{n}^{2}<$ $\zeta_{\min }^{2}=1 / 2$, and for this reason are excluded from the basis. The

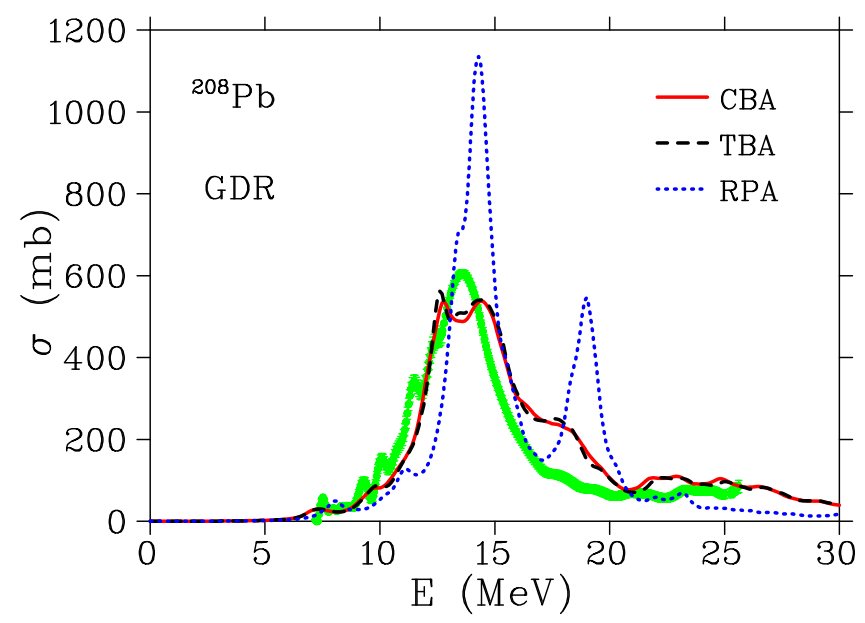

FIG. 6. Same as in Fig. 4 but for ${ }^{208} \mathrm{~Pb}$. Experimental data are taken from Ref. [39].

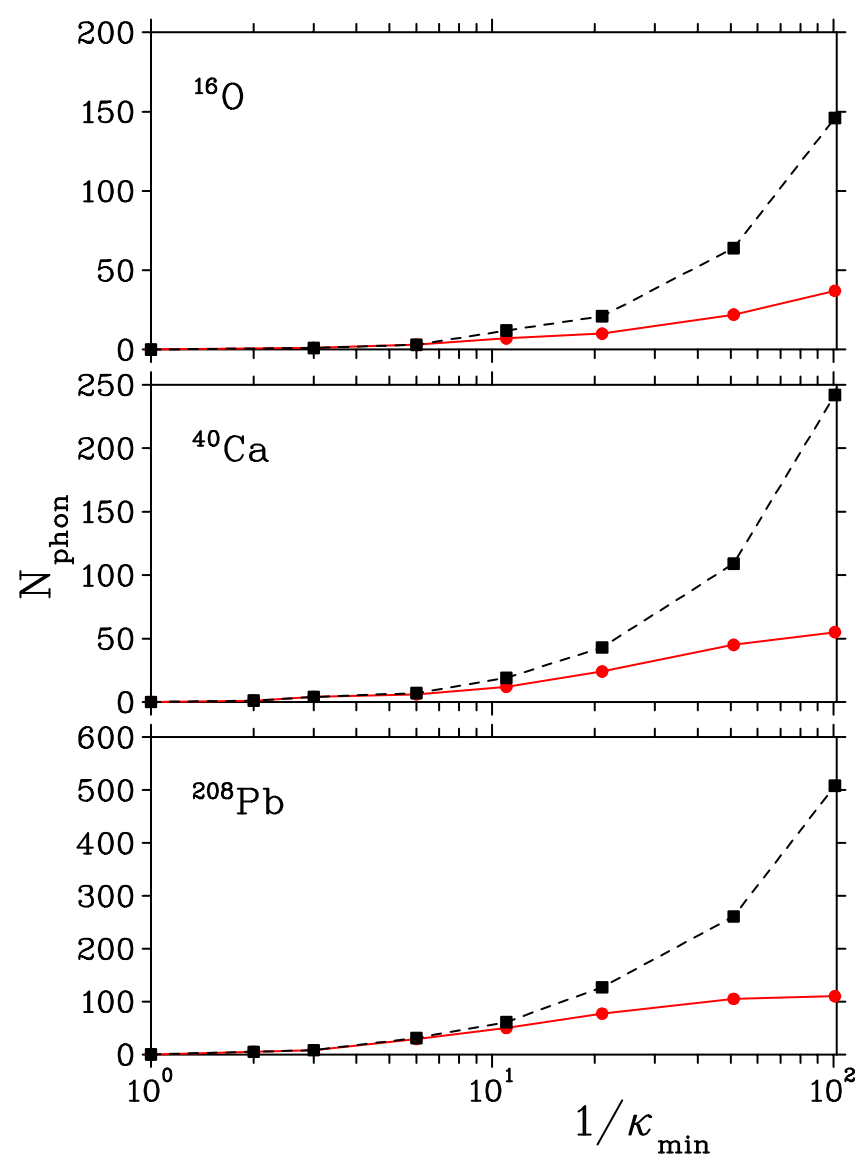

FIG. 7. Dependence of the number of the active TBA phonons in the $\mathrm{CBA}$ with $\zeta_{\mathrm{min}}^{2}=0.5$ (red solid lines) and the number of the RPA phonons in the ordinary TBA (black dashed lines) on the value of the cutoff parameter $\kappa_{\min }$.

value of $\omega_{\max }$ is different for different nuclei. In the calculations presented in Figs. 1-7, it is approximately $30 \mathrm{MeV}$ for ${ }^{16} \mathrm{O}$, $26 \mathrm{MeV}$ for ${ }^{40} \mathrm{Ca}$, and $14 \mathrm{MeV}$ for ${ }^{208} \mathrm{~Pb}$. It is highly satisfying to see that the new selection criterion provides the same cutoff in phonon space as the previous external cutoff criterion developed from the density of phonon states [34], while the new criterion is now inherent in the scheme and so more natural.

The additional effect of the renormalization, Eq. (25), consists in a decrease of the phonon's energies, because, as a rule, $\tilde{\omega}_{n}<\omega_{n}$ for positive $\omega_{n}$. In most cases, this decrease improves the agreement with the experiment. For the nuclei considered in the present work, the calculated and experimental values of the energies of the $3_{1}^{-}$states are listed in Table I. As before, the Skyrme parametrization SV-m64k6 was used. The CBA results were obtained with $\kappa_{\min }=0.01$ and $\zeta_{\min }^{2}=0.5$. The CBA results in the diagonal approximation CBA(D) imply the approximation described in Secs. IID and IIE, that concerns the active TBA phonons used in the CBA (see Sec. IIC). As can be seen from this Table, the diagonal approximation keeps the deviation from the exact results for these states within $0.4 \%$. The central question in our investigation concerns the convergence as a function of the size of the phonon space, and the results are indeed very convincing. 
TABLE I. The energies of the $33_{1}^{-}$states in ${ }^{16} \mathrm{O},{ }^{40} \mathrm{Ca}$, and ${ }^{208} \mathrm{~Pb}$ calculated within the RPA, the CBA, and the CBA in the diagonal approximation $\mathrm{CBA}(\mathrm{D})$. The experimental data are given in the last column.

\begin{tabular}{lcccc}
\hline \hline & \multicolumn{4}{c}{ Energy of the $3_{1}^{-}$state $(\mathrm{MeV})$} \\
\cline { 2 - 5 } & RPA & CBA & CBA(D) & Experiment \\
\hline${ }^{16} \mathrm{O}$ & 8.21 & 7.95 & 7.94 & 6.13 \\
${ }^{40} \mathrm{Ca}$ & 4.00 & 3.84 & 3.83 & 3.74 \\
${ }^{208} \mathrm{~Pb}$ & 3.29 & 3.10 & 3.09 & 2.61 \\
\hline \hline
\end{tabular}

As mentioned above, the value $\zeta_{\min }^{2}=0.5$ is a threshold below which the fragmentation of the RPA state becomes significant. To study the sensitivity of the results to the increase of $\zeta_{\min }^{2}$, we have calculated the energies of the first $3^{-}$levels in ${ }^{16} \mathrm{O},{ }^{40} \mathrm{Ca}$, and ${ }^{208} \mathrm{~Pb}$ in the $\mathrm{CBA}$ with $\zeta_{\min }^{2}$ equal to 0.6 and 0.7 . The results are shown in Figs. 8-10, where the energy differences $\Delta \omega\left(3_{1}^{-}\right)=\omega\left(3_{1}^{-} ; \zeta_{\min }^{2}=0.6\right)-\omega\left(3_{1}^{-} ; \zeta_{\min }^{2}=0.5\right)(\mathrm{red}$ solid lines) and $\Delta \omega\left(3_{1}^{-}\right)=\omega\left(3_{1}^{-} ; \zeta_{\min }^{2}=0.7\right)-\omega\left(3_{1}^{-} ; \zeta_{\min }^{2}=\right.$ $0.5)$ (black dashed lines) are plotted versus the values of $1 / \kappa_{\min }$. As can be seen from Figs. 8 and 9, these energy differences have a tendency to decrease in ${ }^{16} \mathrm{O}$ and ${ }^{40} \mathrm{Ca}$ in the region near $\kappa_{\min }=10^{-3}$. For ${ }^{208} \mathrm{~Pb}$, they are stabilized in the region slightly above $\kappa_{\min }=10^{-2}$. The value of $\Delta \omega\left(3_{1}^{-}\right)$at $\zeta_{\min }^{2}=0.6$ and $\kappa_{\min }=10^{-2}$ amounts to $7 \mathrm{keV}$ for ${ }^{208} \mathrm{~Pb}$ (that corresponds to $4 \%$ of the overall shift of the CBA $3_{1}^{-}$energy with respect to the RPA one). The values of $\Delta \omega\left(3_{1}^{-}\right)$at $\zeta_{\min }^{2}=0.6$ and $\kappa_{\min }=10^{-3}$ amount to $2 \mathrm{keV}$ for ${ }^{40} \mathrm{Ca}$ (1\% of the overall shift) and $22 \mathrm{keV}$ for ${ }^{16} \mathrm{O}(8 \%$ of the overall shift). Thus, one can conclude that the small increase of $\zeta_{\min }^{2}$ with respect to the value $\zeta_{\min }^{2}=0.5$ only slightly affects the results.

\section{CONCLUSIONS}

We have studied phonon-coupling models for nuclear resonances within the time blocking approximation (TBA). A

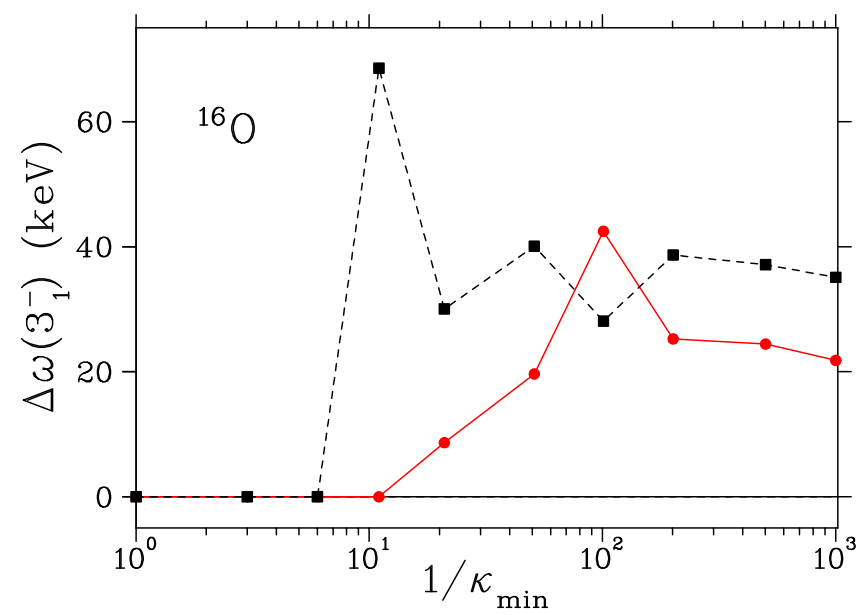

FIG. 8. Differences between the energies of $3_{1}^{-}$state in the nucleus ${ }^{16} \mathrm{O}$ as calculated in the CBA with different values of the cutoff $\zeta_{\min }^{2}$, namely $\Delta \omega\left(3_{1}^{-}\right)=\omega\left(3_{1}^{-} ; \zeta_{\min }^{2}=0.6\right)-\omega\left(3_{1}^{-} ; \zeta_{\min }^{2}=0.5\right)($ red solid lines) and $\Delta \omega\left(3_{1}^{-}\right)=\omega\left(3_{1}^{-} ; \zeta_{\min }^{2}=0.7\right)-\omega\left(3_{1}^{-} ; \zeta_{\min }^{2}=0.5\right)($ black dashed lines).

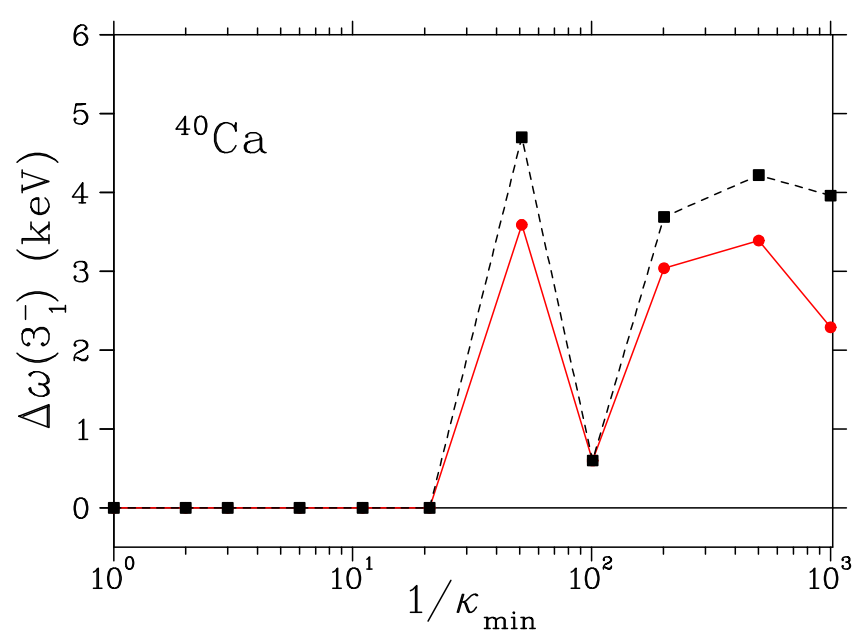

FIG. 9. Same as in Fig. 8 but for the nucleus ${ }^{40} \mathrm{Ca}$.

generalized version of TBA is developed in which the selfconsistency principle is extended to the phonon space of the model. This leads to a nonlinear equation for the energies and transition amplitudes of the nuclear excited states (phonons). The most general version of this nonlinear equation is simplified in two steps: First, the space of phonons to be included in the expansion is limited by the natural requirement that only phonons with dominant $1 \mathrm{p} 1 \mathrm{~h}$ contributions are selected. This is the configuration blocking approximation (CBA), which we use as name for the new scheme. The formalism implies a precise limit that the $1 \mathrm{p} 1 \mathrm{~h}$ content must be larger than $50 \%$. Second, one invokes a diagonal approximation in the representation of the complete set of the solutions of the RPA equations. It turns out that in this diagonal approximation CBA is equivalent to the renormalization of the amplitudes of the phonons entering the phonon basis of the model, which could also describe the new scheme alternatively as a renormalized TBA. The CBA is analyzed in the calculations of the first $3^{-}$ states and the giant dipole resonances in magic nuclei ${ }^{16} \mathrm{O}$, ${ }^{40} \mathrm{Ca}$, and ${ }^{208} \mathrm{~Pb}$. It is shown that CBA produces a natural convergence of the results with respect to enlarging the phonon

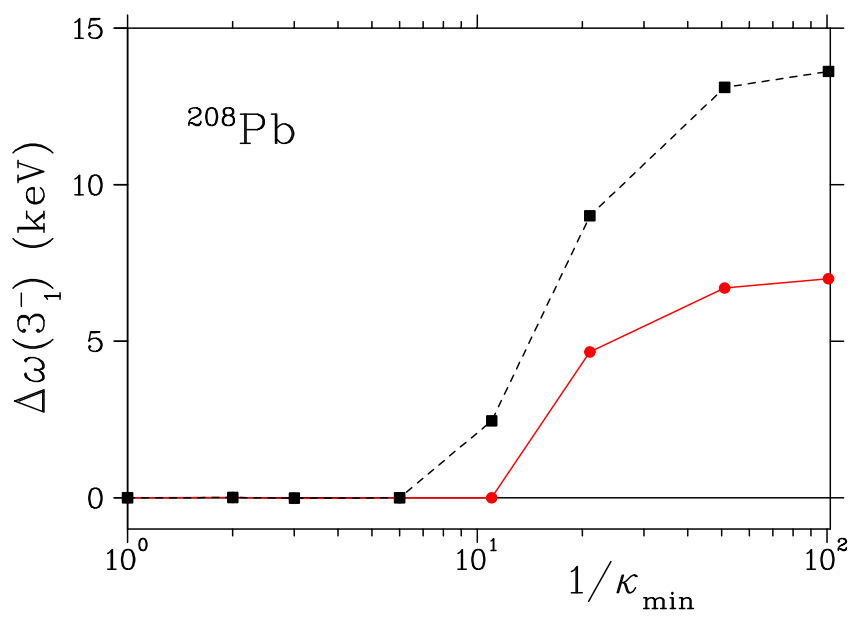

FIG. 10. Same as in Fig. 8 but for the nucleus ${ }^{208} \mathrm{~Pb}$. 
space of the model. This is an advantage as compared to ordinary TBA where additional, external criteria are needed to limit the phonon expansion basis in reasonable manner. This was done previously by determining the cutoff from the density of phonon states. It is highly satisfying that the new, implicit cutoff in CBA produces converged results in agreement with the previous selection scheme.

\section{ACKNOWLEDGMENTS}

V.T. and N.L. acknowledge financial support from the Russian Science Foundation (Project No. 16-12-10155). This research was supported by the Computer Center of St. Petersburg State University. This work was supported by Contract No. Re322-13/1 from the DFG.
[1] A. B. Migdal, Theory of Finite Fermi Systems and Applications to Atomic Nuclei (Interscience, New York, 1967).

[2] J. Speth, E. Werner, and W. Wild, Phys. Rep. 33, 127 (1977).

[3] P. Schuck and S. Ethofer, Nucl. Phys. A 212, 269 (1973).

[4] J. Dukelsky and P. Schuck, Nucl. Phys. A 512, 466 (1990).

[5] J. Dukelsky, G. Röpke, and P. Schuck, Nucl. Phys. A 628, 17 (1998).

[6] P. Schuck and M. Tohyama, Eur. Phys. J. A 52, 307 (2016)

[7] J. D. Immele and G. L. Struble, Phys. Rev. C 15, 1103 (1977).

[8] S. E. Muraviev and M. G. Urin, Fiz. Elem. Chastits At. Yadra 22, 882 (1991) [Sov. J. Part. Nucl. 22, 428 (1991)].

[9] E. Litvinova, Phys. Rev. C 91, 034332 (2015).

[10] S. Drożdż, S. Nishizaki, J. Speth, and J. Wambach, Phys. Rep. 197, 1 (1990).

[11] P. Papakonstantinou and R. Roth, Phys. Lett. B 671, 356 (2009).

[12] D. Gambacurta, M. Grasso, V. De Donno, G. Co', and F. Catara, Phys. Rev. C 86, 021304(R) (2012).

[13] G. F. Bertsch, P. F. Bortignon, and R. A. Broglia, Rev. Mod. Phys. 55, 287 (1983).

[14] C. Barbieri and W. H. Dickhoff, Phys. Rev. C 68, 014311 (2003).

[15] Y. F. Niu, G. Colò, E. Vigezzi, C. L. Bai, and H. Sagawa, Phys. Rev. C 94, 064328 (2016).

[16] V. Tselyaev, N. Lyutorovich, J. Speth, S. Krewald, and P.-G. Reinhard, Phys. Rev. C 94, 034306 (2016).

[17] X. Roca-Maza, Y. F. Niu, G. Colò, and P. F. Bortignon, J. Phys. G: Nucl. Part. Phys. 44, 044001 (2017).

[18] P. F. Bortignon, R. A. Broglia, D. R. Bes, and R. Liotta, Phys. Rep. 30, 305 (1977).

[19] V. Bernard and N. Van Giai, Nucl. Phys. A 348, 75 (1980).

[20] C. Mahaux, P. F. Bortignon, R. A. Broglia, and C. H. Dasso, Phys. Rep. 120, 1 (1985).

[21] G. Colò, H. Sagawa, and P. F. Bortignon, Phys. Rev. C 82, 064307 (2010).

[22] D. Tarpanov, J. Toivanen, J. Dobaczewski, and B. G. Carlsson, Phys. Rev. C 89, 014307 (2014).
[23] D. Tarpanov, J. Dobaczewski, J. Toivanen, and B. G. Carlsson, Phys. Rev. Lett. 113, 252501 (2014).

[24] K. Moghrabi, M. Grasso, X. Roca-Maza, and G. Colò, Phys. Rev. C 85, 044323 (2012).

[25] N. Lyutorovich, V. Tselyaev, J. Speth, S. Krewald, and P.-G. Reinhard, Phys. At. Nucl. 79, 868 (2017).

[26] V. I. Tselyaev, Yad. Fiz. 50, 1252 (1989) [Sov. J. Nucl. Phys. 50, 780 (1989)].

[27] S. P. Kamerdzhiev, G. Ya. Tertychny, and V. I. Tselyaev, Fiz. Elem. Chastits At. Yadra 28, 333 (1997) [Phys. Part. Nucl. 28, 134 (1997)].

[28] S. Kamerdzhiev, J. Speth, and G. Tertychny, Phys. Rep. 393, 1 (2004).

[29] D. J. Rowe, Nuclear Collective Motion (Methuen, London, 1970).

[30] P. Ring and P. Schuck, The Nuclear Many-Body Problem (Springer, New York, 1980).

[31] K. Gütter, P.-G. Reinhard, K. Wagner, and C. Toepffer, Ann. Phys. (N.Y.) 225, 339 (1993).

[32] C. Toepffer and P.-G. Reinhard, Ann. Phys. (N.Y.) 181, 1 (1988).

[33] V. I. Tselyaev, Phys. Rev. C 88, 054301 (2013).

[34] V. Tselyaev, N. Lyutorovich, J. Speth, and P.-G. Reinhard, Phys. Rev. C 96, 024312 (2017).

[35] A. Bohr and B. Mottelson, Nuclear Structure (Benjamin, New York, 1975), Vol. 2.

[36] N. Lyutorovich, V. I. Tselyaev, J. Speth, S. Krewald, F. Grümmer, and P.-G. Reinhard, Phys. Rev. Lett. 109, 092502 (2012).

[37] B. S. Ishkhanov, I. M. Kapitonov, E. I. Lileeva, E. V. Shirokov, V. A. Erokhova, M. A. Elkin, and A. V. Izotova, preprint INP MSU 2002-27/711.

[38] V. A. Erokhova, M. A. Elkin, A. V. Izotova, B. S. Ishkhanov, I. M. Kapitonov, E. I. Lileeva, and E. V. Shirokov, Izv. Ross. Akad. Nauk, Ser. Fiz. 67, 1479 (2003).

[39] S. N. Belyaev, O. V. Vasiliev, V. V. Voronov, A. A. Nechkin, V. Yu. Ponomarev, and V. A. Semenov, Phys. At. Nucl. 58, 1833 (1995). 